\title{
PSI-SERIES OF QUADRATIC VECTOR FIELDS ON THE PLANE
}

\author{
Amadeu Delshams and Arnau Mir
}

\begin{abstract}
Psi-series (i.e., logarithmic series) for the solutions of quadratic vector fields on the plane are considered. Its existence and convergence is studied, and an algorithm for the location of logarithmic singularities is developed. Moreover, the relationship between psi-series and non-integrability is stressed and in particular it is proved that quadratic systems with psi-series that are not Laurent series do not have an algebraic first integral. Besides, a criterion about non-existence of an analytic first integral is given.
\end{abstract}

\section{Introduction}

The study of the representation of the solutions of a system of differential equations and the relationship with the integrability of this system has been considered by many people. This relationship was initiated by Painlevé, who gave a test of integrability based on the search of systems such that all its solutions could be represented as Laurent series of the time parameter [Inc56], [Hil76]. This Painlevé test was later on transformed in an algorithm, the ARS algorithm [ARS78], that has been used succesfully to detect integrable systems (see [RGB89] for a general review).

According to this Painlevé principle, those systems such that all their solutions can be represented in terms of Laurent series, are in fact integrable systems. There are several results confirming this conjecture under some conditions as well as some counterexamples [Gav92], [RGB89], [BPB87].

To be able to deal with more general singularities for the solutions, like logarithmic ones, we consider a generalization of the Laurent series near a singularity $t_{p}$ of a solution $x(t)$, i.e., the so called psi-series:

$$
x(t)=\sum_{n \geq-k} p_{n}\left(\log \left(\frac{1}{\tau}\right)\right) \tau^{n},
$$


where $p_{n}$ are vectors polynomials and $\tau=t-t_{p}$.

This kind of logarithmic expansions for solutions of systems of ordinary differential equations were called psi-series by Hille (see, for instance, [Hil76]), although they had already been studied in the beginning of the century in systems of the form $t d x / d t=f(t, x)$ by Horn [Hor96]. An account of Horn results may be found in [Gav92]. Besides, they have also been considered in several kind of systems by the authors [MD93], and applied to measure the splitting of separatrices of rapidly forced systems [DMS97].

In this paper, we develop the study of the representation of solutions of quadratic systems on the plane. These systems are important for several applications in chemistry, population evolution, plasma physics, ... (see, for instance $[\mathbf{C F}$ 92 $],[\mathbf{H C F} 93],\left[\mathbf{H C F}^{+} \mathbf{9 6}\right]$ for several references and a special study of the so-called Lotka-Volterra systems), and, at the same time, contain already the main features of polynomial systems with more dimensions. Besides, the study of the algebraic integrability of planar polynomial vector fields has been revisited the last years (see [MRT95] for a connection to the existence of isochronous centers).

In section 2, we give conditions for the existence of psi-series for quadratic vector fields on the plane. Thus, Theorem 1 provides a constructive algorithm under a general hypothesis $\mathbf{H 1}$ and a more restrictive hypothesis $\mathbf{H 2}$, both on the quadratic part, which ensure the existence of a formal two-parametric family of psi-series of the form $x(t)=a / \tau+\cdots$. Its convergence, as well as an estimate for the region of convergence is proved in Theorem 7, whose proof is deferred to section 6 . It is worthmentioning that a different point of view to study this kind of representations consists on reducing the quadratic system to a system of the form studied by Horn, and can be found in [Yos83], [Yos83], [Gav88].

The knowledge of the psi-series expansion provides an algorithm to compute numerically the singularities of solutions. Such an algorithm is presented in section 3 and it is illustrated with an example. The relationship between the behaviour of different psi-series of a solution and integrability of the system is also illustrated. In particular, when one considers different singularities of a solution, and as a consequence, different psi-series, the numerical experiments performed in section 3 show that the location of singularities and the behaviour of the free parameter $p$ behave in an irregular way, in contrast with what happens for integrable systems. So it seems that the dynamical information is hidden between the different singularities of a solution (see also [RGB89] for a related discussion), and it remains to give a rigorous criterion along these lines.

The Painlevé principle is checked in section 4, where all integrable 
quadratic systems with a linear center at the origin are displayed. From the study developed there, one gathers that

- the Painlevé test cannot detect quadratic integrable systems if the first integral is a transcendent function,

- all quadratic system with a complete solution in terms of psi-series (not Laurent series) do not have an algebraic first integral,

and as a matter of fact this last result is proved in Theorem 8 of section 4 . According to this result, the existence of logarithmic singularities should be considered not as an obstacle to integrability, but as an obstacle to algebraic non-integrability. In other words, a quadratic vector field on the plane with "genuine" psi-series can still have a transcendent first integral.

Section 5 is devoted to a result about non-existence of such a trascendent first integral. An aside consequence of Theorem 1 is that there exist at least a solution $\varphi(t)$ expressible in terms of Laurent series when hypothesis $\mathbf{H 1}$ holds and hypothesis $\mathbf{H 2}$ fails. In such general situation, the non-existence of an analytic integral near $\varphi(t)$ is proved in Theorem 9. It is worth mentioning that although its proof uses a Ziglin lemma [Zig82], an analytical expression for $\varphi(t)$ is not needed, as happens in the usual applications of Ziglin theory for Hamiltonian systems.

\section{Existence of psi-series}

Let us consider the following differential system:

$$
\left\{\begin{array}{l}
\frac{d x_{1}}{d t}=\sum_{0 \leq i, j \leq 2} a_{i j}^{(1)} x_{1}^{i} x_{2}^{j} \\
\frac{d x_{2}}{d t}=\sum_{0 \leq i, j \leq 2} a_{i j}^{(2)} x_{1}^{i} x_{2}^{j}
\end{array}\right.
$$

which can also be written in vectorial form as

$$
\frac{d x}{d t}=X_{0}+X_{1}(x)+X_{2}(x),
$$

where $X_{j}(x)$ denotes a homogenous polynomial of degree $j$, for $j=$ $0,1,2$.

Let $t_{p}$ a free parameter denoting a (real or complex) singularity of an arbitrary solution $x(t)$ of system (1), and let us look for an expansion of $x(t)$ such that its dominant term is of the form

$$
\left\{\begin{array}{l}
x_{1}(t)=a_{1} \tau^{\alpha_{1}}+\cdots \\
x_{2}(t)=a_{2} \tau^{\alpha_{2}}+\cdots
\end{array}\right.
$$


If we replace expansion (3) into system (1), we notice that, in order to determine the exponents $\alpha_{j}$, only the terms of order 2 have to be considered (equivalently, we could instead look for solutions of the form $x_{1}(t)=a_{1} \tau^{\alpha_{1}}, x_{2}(t)=a_{2} \tau^{\alpha_{2}}$, of the homogeneous quadratic part $d x / d t=X_{2}(x)$ of system (2)). Assuming that both components of $X_{2}(x)$ are not identically zero, we get 3 possible cases:

a) $\alpha_{1}=\alpha_{2}=-1$.

b) $\alpha_{2}=-1$ and $a_{1}$ is the other free parameter besides $t_{p}$. In this case, it is necessary that $a_{20}^{(1)}=a_{02}^{(1)}=a_{20}^{(2)}=a_{11}^{(2)}=0$ and $a_{11}^{(1)} \cdot a_{02}^{(2)} \neq 0$.

c) $\alpha_{1}=-1$ and $a_{2}$ is the other free parameter besides $t_{p}$. In this case, it is necessary that $a_{02}^{(2)}=a_{20}^{(2)}=a_{02}^{(1)}=a_{11}^{(1)}=0$ and $a_{11}^{(2)} \cdot a_{20}^{(1)} \neq 0$.

In general, expansions with non-integer exponents take place in cases b) and c):

b)

$$
x_{1}=a_{1} \tau^{-a_{11}^{(1)} / a_{02}^{(2)}}+\cdots, \quad x_{2}=-\frac{1}{a_{02}^{(2)}} \tau^{-1}+\cdots
$$

c)

$$
x_{1}=-\frac{1}{a_{20}^{(1)}} \tau^{-1}+\cdots, \quad x_{2}=a_{2} \tau^{-a_{11}^{(1)} / a_{20}^{(1)}}+\cdots
$$

Unless the exponent of $\tau$ be an integer number, the singularity $t_{p}$ is a branching point of $x(t)$. If the exponent of $\tau$ is an integer number, the Painlevé property is satisfied [Hil76] and thus we have already the two free parameters $t_{p}$ and $a_{1}$ (or $a_{2}$ ) that provide the general solution of system (1), and the expansion (3) is nothing else but a Laurent series.

It remains to consider case a), the only one with no restrictions. We are going to see under which conditions psi-series appear.

Theorem 1. Assume that the following two hypothesis hold for system (1):

H1 There exists a complex vector $a=\left(\begin{array}{l}a_{1} \\ a_{2}\end{array}\right)$, with both components non-zero such that $X_{2}(a)=-a$.

$\mathbf{H 2}$ If $\{-2, \lambda\}$ are the eigenvalues of $D X_{2}(a)$, then $\lambda$ is a non-negative integer. 
Then, there exists a (formal) two-parametric family of solutions of system (1), formed by psi-series

$$
x(t)=a \tau^{-1}+\sum_{n \geq 0} p_{n}\left(\log \left(\frac{1}{\tau}\right)\right) \tau^{n} .
$$

Remark 2. Hypothesis $\mathbf{H 1}$ and $\mathbf{H 2}$ only affect $X_{2}$. Due to $\mathbf{H 1},-2$ is an eigenvalue of $D X_{2}(a)$ (see equation (5)). In terms of the coefficients of system (1), the other eigenvalue $\lambda$ can be written as

$$
\lambda=\operatorname{div} X_{2}(a)+2=2\left(a_{1} a_{20}^{(1)}+a_{2} a_{02}^{(2)}\right)+a_{11}^{(1)} a_{2}+a_{11}^{(2)} a_{1}+2 .
$$

We note that the matrix $D X_{2}(a)+$ Id and the numbers $-1, \lambda+1$ are called Kowalevski's matrix and Kowalevski's exponents, respectively, by Yoshida [Yos83].

Proof: If we replace the expansion (3) into equation (2) with $\alpha_{1}=$ $\alpha_{2}=-1$, we obtain:

$$
\begin{aligned}
-a \tau^{-2}+\cdots & =X_{0}+X_{1}\left(a \tau^{-1}+\cdots\right)+X_{2}\left(a \tau^{-1}+\cdots\right) \\
& =X_{0}+\tau^{-1} X_{1}(a)+\tau^{-2} X_{2}(a)+\cdots
\end{aligned}
$$

If we consider the coefficients of $\tau^{-2}$, we get the equation for $a$ : $-a=$ $X_{2}(a)$, which, due to hypothesis $\mathbf{H 1}$, has at least a solution with both components different from zero.

Given $\rho$ an arbitrary number, and taking into account that $X_{2}$ is a quadratic vector field, it turns out that $X_{2}(\rho a)=-\rho^{2} a$, for any arbitrary number $\rho$. Differentiating this equation and putting $\rho=1$, we get

$$
D X_{2}(a) a=-2 a,
$$

i.e., $a$ is an eigenvector of $D X_{2}(a)$ of eigenvalue -2 .

Now, it is very convenient to perform a change of time $s=-\log \tau$, or $\tau=e^{-s}$, where $\tau=t-t_{p}$, and a change of variables:

$$
x=e^{s} a+B w,
$$

where $w$ is the new variable, and $B$ is the matrix of eigenvectors of $D X_{2}(a)$ associated to its eigenvalues $-2, \lambda(\lambda \neq-2$ by hypothesis H2). Denoting ${ }^{\prime}=d / d s=-e^{-s} d / d t$, the differential equation for $w$ is: 
(7) $w^{\prime}=\Lambda w-e^{-s} B^{-1} X_{1}(B w)-\frac{1}{2} e^{-s} B^{-1} D_{2}(B w, B w)$

$$
-e^{-s} B^{-1} X_{0}-B^{-1} X_{1}(a)
$$

where $\Lambda$ is the diagonal matrix $\Lambda=B^{-1} D X_{2}(a) B=\left(\begin{array}{rr}-2 & 0 \\ 0 & \lambda\end{array}\right)$, and $D_{2}$ is the constant quadratic form $D_{2}=D^{2} X_{2}\left(e^{s} a\right)=D^{2} X_{2}(0)$.

Introducing $w_{n}=B^{-1} p_{n}$, the psi-series (4) of $x(s)$ takes the form

$$
x(s)=e^{s} a+\sum_{n=0}^{\infty} p_{n}(s) e^{-n s}=e^{s} a+\sum_{n=0}^{\infty} B w_{n}(s) e^{-n s},
$$

and hence, by the change (6), we look for an expansion of $w$ of the form $w(s)=\sum_{n>0} w_{n}(s) e^{-n s}, w_{n}(s)$ being polynomials in the variable $s$. Since $w^{\prime}=\sum_{n=0}^{\infty}\left(w_{n}^{\prime}-n w_{n}\right) e^{-n s}$, we get the differential equation for $w_{0}, w_{1}$ and $w_{n}$, for $n>1$ :

$$
\begin{aligned}
& w_{0}^{\prime}=-\Lambda w_{0}-e^{-s} B^{-1} X_{0} \\
& w_{1}^{\prime}=-(\Lambda-\mathrm{Id})-B^{-1} X_{1}\left(B w_{0}\right)-\frac{1}{2} B^{-1} D_{2}\left(B w_{0}, B w_{0}\right)-B^{-1} X_{1}(a), \\
& w_{n}^{\prime}=-(\Lambda-n \mathrm{Id}) w_{n}-B^{-1} X_{1}\left(B w_{n-1}\right)-\frac{1}{2} B^{-1} \sum_{k=0}^{n-1} D_{2}\left(B w_{k}, B w_{n-k-1}\right),
\end{aligned}
$$

which can be written in more compact form as

$$
w_{n}^{\prime}=\Lambda_{n} w_{n}+c_{n}
$$

where $\Lambda_{n}=n \operatorname{Id}-\Lambda$ and $c_{n}$ is a polynomial of degree two in the variables $w_{0}, \ldots, w_{n-1}$. The next step is to obtain the polynomial vectors $w_{n}(s)$.

If $n<\lambda, \Lambda_{n}$ is an invertible matrix and the only polynomial solution of the linear system (9) is the constant vector $w_{n}=-\Lambda_{n}^{-1} c_{n}$. So, $p_{n}=B w_{n}$ is constant, too.

If $n=\lambda$, the matrix $\Lambda_{n}$ is singular and it has two eigenvalues: $n+2$ and 0 . In this case, system (9) takes the form

$$
\left\{\begin{array}{l}
w_{n, 1}^{\prime}=(n+2) w_{n, 1}+c_{n, 1} \\
w_{n, 2}^{\prime}=c_{n, 2}
\end{array}\right.
$$

and its only polynomial solution is given by $w_{n, 1}=-c_{n, 1} /(n+2), w_{n, 2}=$ $c_{n, 2} s+c$. The degree of $w_{n}$ and $p_{n}$ depends on $c_{n, 2}$ :

a) If $c_{n, 2}=0, w_{n}$ and $p_{n}$ are constant.

b) If $c_{n, 2} \neq 0, w_{n}$ and $p_{n}$ are polynomials of degree 1 in the variable $s$. 
If $n>\lambda$, in case a), $p_{n}$ is a constant for all $n$ because $\Lambda_{n}$ is a nonsingular matrix for $n>\lambda$. Under these circumstances, the expansions of $x_{1}(t)$ and $x_{2}(t)$ are Laurent series where the other free constant is $c$.

In case b) we use the following simple lemma, whose elementary proof is omitted.

Lemma 3. Let $d y / d s=y^{\prime}=k y+q_{m}(s)$ be a differential equation of first order, where $k$ is a non-zero constant and $q_{m}(s)=\sum_{i=0}^{m} \alpha_{i} s^{i}$ is a polynomial of degree $m$. Then, there exists a unique polynomial solution $y=p_{m}(s)=\sum_{i=0}^{m} \beta_{i} s^{i}$, and its coefficients are given by $\beta_{m}=-\alpha_{m} / k$, $\beta_{i}=\left((i+1) \beta_{i+1}-\alpha_{i}\right) / k,(i=m-1, \ldots, 0)$.

$$
\left(\text { In fact } \beta_{i}=-\sum_{j=0}^{m-i}(i+1) \cdots(i+j) \alpha_{i+j} / k^{j+1}\right. \text {.) }
$$

Applying this lemma to equation (9), and recalling that $c_{n}$ is a polynomial of degree two in the variables $w_{0}, \ldots, w_{n-1}$, it follows that $w_{n}$ is a polynomial of degree $[n / \lambda]$ and therefore genuine psi-series appear.

Remark 4. The coeficient $c_{\lambda, 2}$ could be considered as a coefficient of non-integrability, because when it is zero, there is a biparametric family of meromorphic solutions of system (1), and according to the Painlevé principle, it is a candidate to be an integrable system. We will see in section 4 that $c_{\lambda, 2}$ is in fact a coefficient of algebraic non-integrability.

Remark 5. Hypothesis $\mathbf{H 1}$ is very general, since it is equivalent to the existence of a complex solution $b=\left(\begin{array}{l}b_{1} \\ b_{2}\end{array}\right)$ of $\operatorname{det}\left(X_{2}(b) b\right)=0$, with $b_{1} \cdot b_{2} \neq 0$. Indeed, such $b$ would satisfy $X_{2}(b)=\rho b$ for some complex number $\rho$, and $a=\sqrt{-1 / \rho} \cdot b$ satisfies hypothesis H1. When it fails, other leading terms different from $x(t)=a / \tau+\cdots$ can take place (see, for instance, systems (11) and (15)). Only for the sake of brevity, a general classification of such cases is not considered in this paper.

Remark 6. Hypothesis $\mathbf{H 2}$ imposes severe restrictions on $X_{2}$, since it is required that $\lambda:=\operatorname{div} X_{2}(a)+2 \in\{0,1,2, \ldots\}$. When it fails, one can only assert the existence of one meromorphic solution of system (1). However, we will see in section 5 that the general case $\lambda \notin \mathbb{Q}$ gives rise to analytic non-integrability near this meromorphic solution.

\subsection{Convergence of the psi-series.}

Once the existence of the formal expansion of psi-series has been obtained, it remains the problem of the convergence of such expansions. 
The following theorem gives us a real region, depending on a parameter $K$, where the psi-series are convergent, in terms of the variable $s$. In the sequel, given a vector $x$ and a $p$-linear application $A,\|x\|$ will denote a vectorial norm for $x$, and $\|A\|=\max \left\{\left\|A\left(x^{(1)}, \ldots, x^{(p)}\right)\right\|:\left\|x^{(j)}\right\| \leq 1\right.$, $j=1, \ldots, p\}$ the subordinate $p$-linear norm.

Theorem 7. Consider the constant $C=M\left(\left\|X_{1}\right\|+(\pi / 2)\left\|D_{2}\right\| \cdot\|B\|\right)$, where $B$ is the matrix of eigenvectors of $D_{2}=D^{2} X_{2}(0)$ and $M$ is the condition number of $B: M=\|B\| \cdot\left\|B^{-1}\right\|$.

Let $K>0$ such that the following finite number of inequalities are satisfied:

$\left\|w_{n}(s)\right\| \leq(n+1)^{-1 / 2}(2 K+K s)^{n / \lambda} \quad n=0,1, \ldots, \max \left\{4 \lambda, 9 C^{2}+1\right\}$.

Then, the psi-series $\sum_{n \geq 0} w_{n}(s) e^{-n s}, \sum_{n \geq 0} p_{n}(s) e^{-n s}$ are convergent for $s>$ $s_{0}$, where $s_{0}$ is the positive root of the equation $K(2+s)=e^{\lambda s}$.

The convergence follows from the general theory of analytic ordinary differential equations of the form $t d x / d t=f(t, x), x \in \mathbb{R}^{n}$ which was developed by Horn [Hor96]. A well-adapted proof for quadratic vector fields on the plane can be found in [Hil74], [Smi75]. However, since the estimates over the region of convergence stated above are relevant for the numerical method of the next section, its proof is presented in section 6 .

\section{Numerical location of singularities}

The problem is the following: Let $\left(x_{0}, y_{0}\right)$ be initial conditions of system (1). We want to find a singularity $t_{p}$ and also the other free parameter $c$ of the expansion in psi-series or Laurent series.

We solve numerically the problem in two steps.

\section{Step 1}

The singularities can be of three types:

a) Poles. The expansion is a Laurent series. To locate the poles, we have to find the expansion series in a Taylor series in two points. The convergence regions are two discs. One of the intersection points of these two discs is a pole (see Figure 1) and there is a well known algorithm to compute it due to Chang and Corliss [CC80]. 


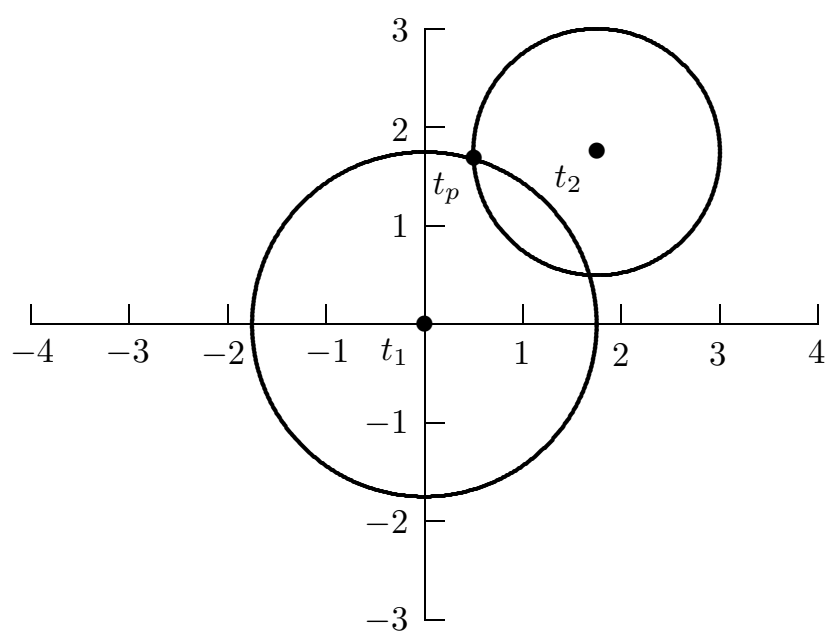

Figure 1. Singularity of pole type.

b) Singularities with rational branching

$$
x\left(t-t_{p}\right)=x(\tau)=\frac{a_{1}}{\tau^{\alpha}}+\sum_{n \geq 0} a_{n} \tau^{\alpha+n \beta}, \text { where } \alpha, \beta \in \mathbb{Q}^{+} .
$$

c) Singularities such that the expansion is a psi-series

$$
x\left(t-t_{p}\right)=x(\tau)=\sum_{n \geq-k} p_{n}\left(\log \frac{1}{\tau}\right) \tau^{n}, \text { where } p_{n} \text { are polynomials. }
$$

To locate singularities of b) or c) type we consider closed paths on the complex plane time. If the value of the solution $x(t)$ of system (1) at the beginning of the path is different from its value at the end of the path, we can deduce that there exists at least a singularity inside the path.

These paths can be chosen as rectangles. Thus, if we take smaller rectangles every time, we can find a first approximation of $t_{p}$ (see Figure 2). There is a difference between the singularities of b) and c) type. The convergence region of the expansions of the singularities of b) type is a Riemann surface with a finite number of sheets whereas the expansions of the singularities of c) type is a Riemann surface with an infinite number of sheets.

So, to know the type of singularity, we turn round it a big enough number of times. If the value of solution $\left(x_{1}(t), x_{2}(t)\right)$ is the same at the end and at the beginning of the path, we have a singularity of b) type; 
otherwise, we have a singularity of c) type. The number of turns for the singularities of $\mathrm{b}$ ) type is the denominator of $\beta$.

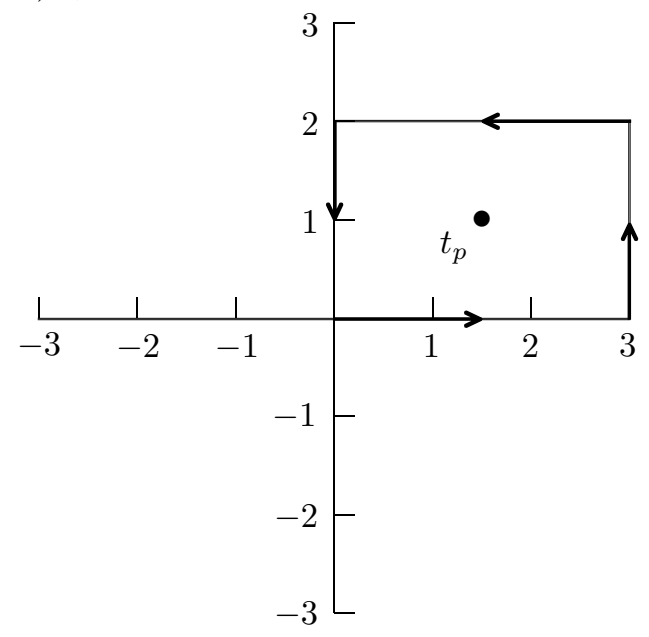

Figure 2. Closed paths to find a singularity.

\section{Step 2}

Using step 1 , we can locate the singularity with 1 or 2 correct digits. Our aim is to find $t_{p}$ with the working precision of the computer. To do it, we choose a fixed complex time $t$ in the region of convergence and find numerically $\left(x_{1}(t), x_{2}(t)\right)$ by using a standard integration method (for instance, we have used Runge-Kutta 7-8), from initial conditions $\left(x_{0}, y_{0}\right)$. Next, we introduce $\Delta t=t-t_{p}$ and we consider the following application (we identify $\mathbb{C}^{2} \equiv \mathbb{R}^{4}$ ):

$$
\begin{aligned}
F_{\Delta t}: \quad \mathbb{R}^{4} & \longrightarrow \mathbb{R}^{4} \\
\left(t_{p}, c\right) & \longmapsto\left(x_{1}(t), x_{2}(t)\right)=a / \Delta t+\sum p_{n}(\Delta t)^{n} .
\end{aligned}
$$

So, we have to solve numerically the equation $F_{\Delta t}\left(t_{p}, c\right)=\left(x_{1}(t), x_{2}(t)\right)$ in $\mathbb{R}^{4}$. To such purpose we use, for example, the Newton-Raphson method, with the initial approximation of $t_{p}$ from the step 1 . The initial approximation of $c$ is not important because it appears in a term of higher order. So, we fix $\left(t_{p_{0}}, c\right)$ and we only have to repeat this process:

$$
\left(\begin{array}{c}
t_{p} \\
c
\end{array}\right)_{n+1}=\left(\begin{array}{c}
t_{p} \\
c
\end{array}\right)_{n}-\left(D F_{\Delta t}\left(\left(\begin{array}{c}
t_{p} \\
c
\end{array}\right)_{n}\right)\right)^{-1}\left(F_{\Delta t}\left(\left(\begin{array}{c}
t_{p} \\
c
\end{array}\right)_{n}\right)-\left(\begin{array}{c}
x_{1}(t) \\
x_{2}(t)
\end{array}\right)\right) .
$$




\subsection{An example.}

We consider the following system of differential equations depending on the parameters $\epsilon$ and $\gamma$ :

$$
\left\{\begin{array}{l}
\dot{x}=y \\
\dot{y}=-x+\gamma x^{2}+y^{2}+\epsilon x y
\end{array}\right.
$$

For $\epsilon=0$, this system is integrable system with general integral:

$$
e^{-2 x}\left(2 y^{2}+2(\gamma-1) x+2 \gamma x^{2}+\gamma-1\right)=C .
$$

and has a center at the origin. This center dissapears for $\epsilon \neq 0$ (the origin is a repulsor point for $\epsilon>0$ ), and system (11) turns out to be non-integrable. In fact, in section 4 , all the integrable quadratic systems with a (linear) center at the origin will be classified, and system (11) is named as case III of Table 3 for $\epsilon=0$; for $\epsilon \neq 0$, system (11) is different from all the integrable cases therein.

The psi-series expansions of $x(t)$ and $y(t)$ are given by

$$
\begin{aligned}
& x(t)=-\log \tau+p+[-(3+2 p) \epsilon / 4+(\epsilon / 2) \log \tau] \tau \\
& +\left[\frac{1}{864}\left(-120-144 p+139 \epsilon^{2}+12 p \epsilon^{2}-36 p^{2} \epsilon^{2}+152 \gamma+240 p \gamma+144 p^{2} \gamma\right)\right. \\
& \left.-\frac{1}{72}\left(-12+\epsilon^{2}-6 p \epsilon^{2}+20 \gamma+24 p \gamma\right) \log \tau+\left(-\frac{\epsilon^{2}}{24}+\frac{\gamma}{6}\right)(\log \tau)^{2}\right] \tau^{2} \\
& +\mathrm{O}\left(\tau^{3}(\log \tau)^{3}\right), \\
& y(t)=-1 / \tau+(-(1+2 p) \epsilon / 4+(\epsilon) / 2 \log \tau) \\
& +\left[\frac{1}{432}\left(-48-144 p+133 \epsilon^{2}+48 p \epsilon^{2}-36 p^{2} \epsilon^{2}+32 \gamma+96 p \gamma+144 p^{2} \gamma\right)\right. \\
& \left.-\frac{1}{18}\left(-6+2 \epsilon^{2}+3 p \epsilon^{2}+4 \gamma+12 p \gamma\right) \log \tau+\left(-\epsilon^{2} / 12+\gamma / 3\right)(\log \tau)^{2}\right] \tau \\
& +\left[\frac{\epsilon}{3456}\left(996+720 p+19 \epsilon^{2}+516 p \epsilon^{2}+288 p^{2} \epsilon^{2}-736 \gamma-2208 p \gamma-1152 p^{2} \gamma\right)\right. \\
& \left.-\frac{\epsilon}{288}\left(60+43 \epsilon^{2}+48 p \epsilon^{2}-184 \gamma-192 p \gamma\right) \log \tau+\frac{1}{12}\left(\epsilon^{3}-4 \epsilon \gamma\right)(\log \tau)^{2}\right] \tau^{2} \\
& +\mathrm{O}\left(\tau^{3}(\log \tau)^{4}\right),
\end{aligned}
$$

where $\tau=t-t_{p}$ and $p$ is the other free parameter.

We note that the expansion of $x(t)$ begins with a term of "log $\tau$ " type instead of a term of pole type, and therefore it is very difficult to find such singularities with the standard methods. Following the method described 
at the beginning of this section, we have chosen initial conditions near the origin: $x_{0}=0.1$ and $y_{0}=0$, and we have found the singularities $t_{p}$ in the strip $-3 \leq \Re t_{p} \leq 3$ and $\Im t_{p}>0$ that are closest to the real axis. These singularities, as well as the value of the parameter $p$ and the radius of convergence $R_{\text {conv }}$ (i.e., the radius of the biggest circle included in the region of convergence of the expansion), are shown in Table 1 for several values of $\epsilon$. A more complete picture of the singularities, for $\epsilon=0$ and $\epsilon=0.1$ is shown in Figure 3 .

\begin{tabular}{|c|c|c|c|c|c|}
\hline$\epsilon$ & $\Re t_{p}$ & $\Im t_{p}$ & $\Re p$ & $\Im p$ & $R_{\mathrm{conv}}$ \\
\hline 0. & -0.466299 & 4.726057 & 2.402585 & 0. & 0.8 \\
\hline 0.1 & -0.751200 & 4.801549 & 1.655067 & 0.558474 & 0.9 \\
\hline 0.2 & -0.837367 & 4.878813 & 1.488597 & 0.489605 & 1.0 \\
\hline 0.3 & -0.896120 & 4.979702 & 1.480663 & 0.402340 & 1.0 \\
\hline 0.4 & -0.945307 & 5.098131 & 1.553851 & 0.310728 & 1.0 \\
\hline 0.5 & -0.991941 & 5.234179 & 1.688441 & 0.215677 & 1.0 \\
\hline 0.6 & -1.041490 & 5.390227 & 1.882436 & 0.117674 & 1.0 \\
\hline 0.7 & -1.101068 & 5.569672 & 2.143617 & 0.021062 & 0.9 \\
\hline 0.8 & -1.182118 & 5.774325 & 2.484543 & -0.058880 & 0.8 \\
\hline 0.9 & -1.299707 & 5.997159 & 2.904342 & -0.090138 & 0.7 \\
\hline 0. & 0.466299 & 4.726057 & 2.402585 & 3.141592 & 0.8 \\
\hline 0.1 & 0.467743 & 4.278567 & 1.446336 & 3.716525 & 0.9 \\
\hline 0.2 & 0.556347 & 4.082253 & 1.053544 & 3.677679 & 0.9 \\
\hline 0.3 & 0.624368 & 3.943906 & 0.802739 & 3.629956 & 1.0 \\
\hline 0.4 & 0.679105 & 3.832637 & 0.612744 & 3.586886 & 1.0 \\
\hline 0.5 & 0.724686 & 3.737782 & 0.457327 & 3.549249 & 1.0 \\
\hline 0.6 & 0.763537 & 3.654240 & 0.324603 & 3.516411 & 1.0 \\
\hline 0.7 & 0.797211 & 3.579117 & 0.208124 & 3.487626 & 1.0 \\
\hline 0.8 & 0.826771 & 3.510585 & 0.103966 & 3.462242 & 1.1 \\
\hline 0.9 & 0.852979 & 3.447400 & 0.009547 & 3.439724 & 1.1 \\
\hline
\end{tabular}

Table 1 . Singularity $t_{p}$, free parameter $p$ and radius of convergence $R_{\text {conv }}$ of system (11) with $\gamma=1$, for initial conditions $x_{0}=0.1, y_{0}=0$. 
We finish this example by showing in Figure 4 the evolution of $\Re t_{p}$ and $\Im t_{p}$ of the singularity nearest to the real axis, for $\Im t_{p}>0$ and $\Re t_{p}>0$, as a function of $\epsilon$. We observe that in the non-integrable case, the singularities look closer to each other than in the integrable case. We also observe that in the integrable case, the difference between two free constants $p$ of two "consecutive" singularities is the same but this is not longer true in the non-integrable case.
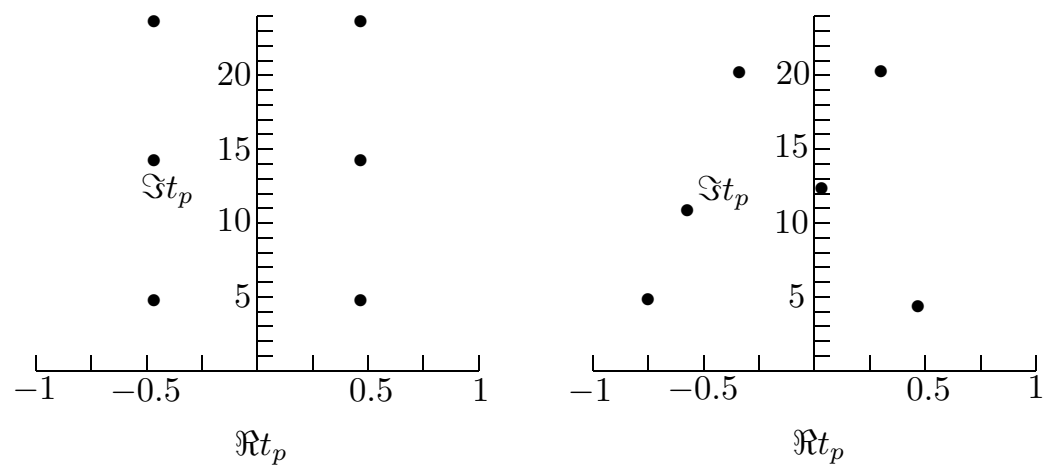

Figure 3. Location of singularities of system (11) for $\gamma=1$, and initial conditions $x_{0}=0.1, y_{0}=0 . \epsilon=0$ in the left figure, and $\epsilon=0.1$ in the right one.

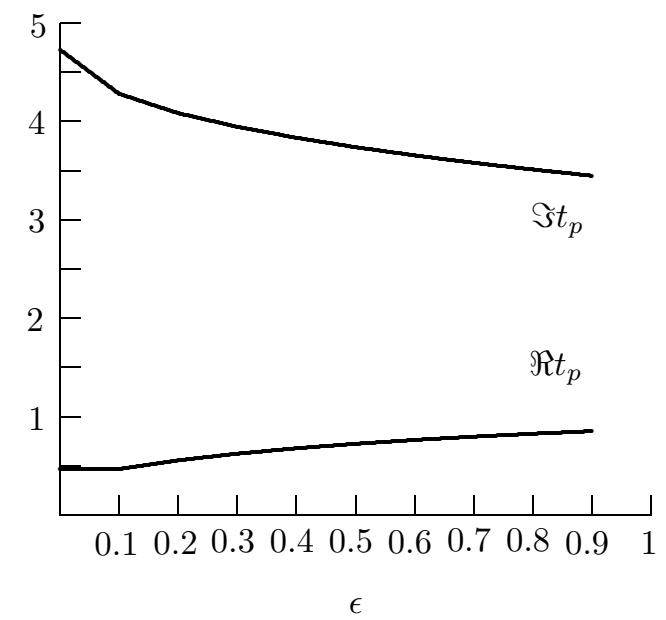

Figure 4. Evolution of $\Re t_{p}$ and $\Im t_{p}$ of one singularity of system (11) for $\gamma=1$ and initial conditions $x_{0}=0.1, y_{0}=0$. 


\begin{tabular}{l|r|l|r|r|l}
$\epsilon$ & $\Re t_{p}$ & \multicolumn{1}{|c}{$\Im t_{p}$} & $\Re p$ & $\Im p$ & $R_{\text {conv }}$ \\
\hline 0. & 0.871592 & 0. & 0.653426 & 3.141592 & 1.5 \\
0. & -0.359139 & 2.789944 & 0.653426 & 6.283185 & 0.7 \\
0. & -0.633889 & 3.405586 & 0.653426 & 9.424777 & 0.6 \\
0. & -0.755672 & 3.784135 & 0.653426 & 12.566370 & 0.4 \\
\hline 0.1 & 0.810223 & 0. & 0.648995 & 2.855993 & 1.6 \\
0.1 & -0.324740 & 2.801453 & 0.648995 & 5.711986 & 0.8 \\
0.1 & -0.607142 & 3.450232 & 0.648995 & 8.567979 & 0.6 \\
0.1 & -0.732948 & 3.848095 & 0.648995 & 11.423973 & 0.5
\end{tabular}

Table 2. Singularities $t_{p}$, free parameter $p$ and radius of convergence $R_{\text {conv }}$ of system (11) with $\gamma=1$, for initial conditions $x_{0}=0.1, y_{0}=0$.

The location of this free parameter $p$ seems to be important for the integrability of a system. To ilustrate this behaviour, in Table 2 is shown this constant difference of two "consecutive" singularities for the system

$$
\left\{\begin{array}{l}
\dot{x}=y \\
\dot{y}=-x+\gamma x^{2}+(1+\epsilon) y^{2}
\end{array}\right.
$$

which has general integral

$$
2(1+\epsilon)^{2} y^{2}+2(\gamma-1-\epsilon) x+2 \gamma(1+\epsilon) x^{2}+\frac{\gamma-1-\epsilon}{1+\epsilon}=C e^{2(1+\epsilon) x} .
$$

\section{Psi-series and integrability}

In this section, we are going to find out which is the relation between the existence of psi-series and non-integrability. To this end, we first will try to see if psi-series (not reducible to Laurent series) can appear in integrable systems. To test this possibility we consider all the quadratic integrable systems

$$
\left\{\begin{array}{l}
\dot{x}=a x+a_{20} x^{2}+a_{11} x y+a_{02} y^{2}, \\
\dot{y}=b y+b_{20} x^{2}+b_{11} x y+b_{02} y^{2},
\end{array}\right.
$$

with a linear center at the origin. These systems were determined by Lunkevich and Sibirskii [LS82], and are shown in Table 3. 


\begin{tabular}{|c|c|c|}
\hline Case & Differential system & General Integral \\
\hline I & $\begin{aligned} \dot{x}= & y+a_{20} x^{2} \\
& +2 a_{11} x y+a_{02} y^{2} \\
\dot{y}= & -x+b_{20} x^{2} \\
& -2 a_{20} x y-a_{11} y^{2}\end{aligned}$ & $\begin{array}{r}3\left(x^{2}+y^{2}\right)-2\left(b_{20} x^{3}-3 a_{20} x^{2} y\right. \\
\left.-3 a_{11} x y^{2}-a_{02} y^{3}\right)=C\end{array}$ \\
\hline II & $\begin{array}{r}\dot{x}=y+2 \alpha x y \\
\dot{y}=-x+\gamma x^{2}+(1-\alpha) y^{2} \\
\alpha \neq 0,1 / 3,1 / 2,1\end{array}$ & $\begin{array}{l}(1+2 \alpha x)^{\alpha-1} \cdot(\gamma+3 \alpha-1 \\
+2(\alpha-1)(2 \alpha-1)\left(\gamma x^{2}-(3 \alpha-1) y^{2}\right) \\
\quad-2(\alpha-1)(\gamma+3 \alpha-1) x)^{\alpha}=C\end{array}$ \\
\hline III & Case II with $\alpha=0$ & $2 y^{2}+2(\gamma-1) x+2 \gamma x^{2}+\gamma-1=C e^{2 x}$ \\
\hline IV & Case II with $\alpha=1$ & $4 y^{2}-2 \gamma x^{2}+2(\gamma+2) x=(\gamma+2) \ln |1+2 x|+C$ \\
\hline $\mathrm{V}$ & Case II with $\alpha=1 / 2$ & $\frac{2 \gamma x(1+x)-y^{2}-2(\gamma+1)}{2(x+1)}=(2 \gamma+1) \ln |x+1|+C$ \\
\hline VI & Case II with $\alpha=1 / 3$ & $\frac{4 y^{2}+6 \gamma x^{2}-6(3 \gamma+2) x-9(3 \gamma+1)}{3(3+2 x)^{2}}=\gamma \ln |2 x+3|+C$ \\
\hline VII & $\begin{aligned} & \dot{x}= y+\gamma x^{2} \\
&+\left(3+\gamma^{2}\right) x y-\gamma y^{2} \\
& \dot{y}=-x-\gamma x^{2}-2 \gamma x y \\
&+\left(1+2 \gamma^{2}\right) y^{2} \\
& \gamma \neq 0\end{aligned}$ & $\begin{aligned} \phi_{1}^{\beta_{1}} \cdot \phi_{2}^{\beta_{2}}=C, \text { where } & \\
\phi_{1}= & 1+\left(1+\gamma^{2}\right) \\
& \cdot\left(3 x+3 \gamma x(y+\gamma x)+\left(\gamma(y+\gamma x)^{3}\right)\right. \\
\phi_{2}= & 1+\left(1+\gamma^{2}\right)\left(2 x+(y+\gamma x)^{2}\right) \\
\beta_{1}= & -2\left(1+\gamma^{2}\right)^{-1} / 3, \quad \beta_{2}=\left(1+\gamma^{2}\right)^{-1}\end{aligned}$ \\
\hline VIII & $\begin{array}{l}\dot{x}=y-\left(1+b^{2}\right) x y \\
\dot{y}=-x+x^{2}+2 b x y-y^{2}\end{array}$ & $\begin{array}{l}(1-x-2 b y)(1-x)^{-\left(1+2 b^{2}\right)} \\
=C \exp (2 b(2 b-y) /(1-x))\end{array}$ \\
\hline IX & $\begin{array}{c}\dot{x}=y+(1-c) x y \\
\dot{y}=-x+c x^{2} \\
+2 b x y-c y^{2} \\
\quad c \neq 0,1\end{array}$ & 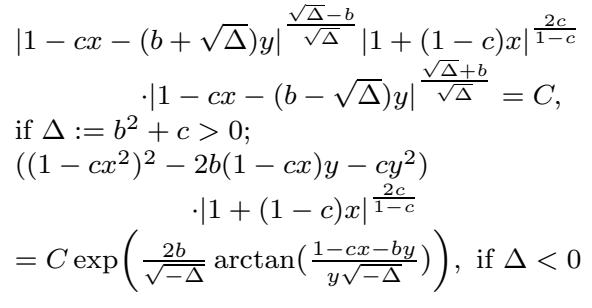 \\
\hline $\mathrm{X}$ & Case IX with $c=0,1$ & $\begin{array}{r}\mid 1-x-\left(b+\left.\sqrt{b^{2}+1} y\right|^{b-\sqrt{b^{2}+1}}\right. \\
\cdot \mid 1-x-\left(b-\left.\sqrt{b^{2}+1} y\right|^{-\left(b-\sqrt{b^{2}+1}\right.}\right) \\
=C \exp \left(2 x \sqrt{b^{2}+1}\right)\end{array}$ \\
\hline
\end{tabular}

Table 3. Quadratic integrable systems. 
For each system that appears in Table 3, we have checked if hypothesis $\mathbf{H 1}$ and $\mathbf{H 2}$ of Theorem 1 are verified. If this is the case, we put YES in the column "psi-series" of Table 4, we write $\lambda+1$ and we check if the psi-series are in fact Laurent series.

\begin{tabular}{c|c|c|c} 
Case & $\lambda+1$ & Psi-series & Laurent series \\
\hline I & 3 & YES & YES \\
II & $3-1 / \alpha$ & YES if $\alpha=-\frac{1}{n}, n=1,2, \ldots$ & YES for $n=1,2, \ldots, 7$ \\
III & 0 & YES & NO \\
IV & 2 & YES & NO $($ YES if $\gamma=-2)$ \\
V & 1 & YES & NO $($ YES if $\gamma=-1 / 2)$ \\
VI & $?$ & NO & NO \\
VII & $1 / 2,-2$ & YES & YES \\
VIII & 0 & YES & YES \\
IX & $-\frac{2(\Delta \pm b \sqrt{\Delta})}{c(1-c)}$ & YES if $r=1,2, \ldots$ & YES \\
X & 1 & YES & NO
\end{tabular}

Table 4. Occurrence of psi- and Laurent series.

In cases I, II and $\alpha=1 / n$, IV and V, Laurent series appear only when the general integral $H(x, y)$ is an algebraic function: $p(x, y, H)=0$, where $p$ is a polynomial in its three variables $x, y, H$. This phenomenum also takes place in case IX, but it requires more cumbersome computations.

In view of this fact, we can conjecture that for the algebraically integrable systems, psi-series reduce to Laurent series. This is confirmed by cases III and X where psi-series are not Laurent series, and the integrals are not algebraic. We notice that the reciprocal assertion is false, since system VIII, for integer $b^{2}+1$, is not algebraically integrable and only Laurent series appear.

It is worth mentioning that the Painlevé test cannot detect integrable systems such that the first integral is not algebraic. For instance, case III cannot be detected by this test.

System VI is a special one, in the sense that standard psi-series do not appear, but instead one can find the following expansions, where $\beta$ 
is the other free parameter,

$$
\begin{aligned}
& x(t)= \pm(\log \tau)^{-1 / 2} \tau^{-1}+\beta(\log \tau)^{-3 / 2} \tau^{-1}+\cdots, \\
& y(t)=-\frac{3}{2} \tau^{-1}-\frac{3}{4}(\log \tau)^{-1} \tau^{-1}+\cdots
\end{aligned}
$$

which can be seen as generalized psi-series.

After all the above motivation, we are now in position to prove that quadratic systems on the plane with psi-series not reducible to Laurent series cannot have an algebraic first integral. This result was also proved by Gavrilov [Gav88], but the proof that we present here is immediate thanks to the framework introduced in the proof of Theorem 1. It is worth mentioning here that the proof of Theorem 1 provides us with an algorithm to detect the existence of genuine psi-series, and therefore, in view of next theorem, of an obstruction to the existence of algebraic first integrals.

Theorem 8. Assume that system (1) has a non-trivial algebraic integral:

$$
p(x, H)=0,
$$

where $p$ is a non-constant polynomial in the variable $x$ and the constant of integration $H$. Then, if hypothesis $\mathbf{H 1}, \mathbf{H 2}$ hold, the psi-series provided by Theorem 1 are Laurent series.

Proof: Consider the change of time $s=-\log \tau$ and the change of variables $(6)$

$$
x=e^{s} a+B w,
$$

as in section 2 , and let $m$ be the degree of the polynomial $p$.

If we expand the first integral $p\left(e^{s} a+B w, H\right)=0$ of system (7) in Taylor series with respect to $w$, we get:

$$
\begin{aligned}
p\left(e^{s} a+B w, H\right)= & p\left(e^{s} a, H\right)+D p\left(e^{s} a, H\right) B w \\
& +\frac{1}{2} D^{2} p\left(e^{s} a, H\right)(B w, B w) \\
& \left.+\frac{1}{m !} D^{m} p\left(e^{s} a, H\right)(B w, . m), B w\right)=0 .
\end{aligned}
$$

Substituting the expansion $w=\sum_{n>0} w_{n} e^{-n s}$, in the previous formula, and equating to zero the coefficients of $e^{-n s}$, we arrive at an expression of the form:

$$
p_{n}\left(w_{n}, w_{n-1}, \ldots, w_{0}, H\right)=0,
$$


where $p_{n}$ is a polynomial in the variables $w_{i}, i=0, \ldots, n$ and $H$, which is a first integral of the equation (10), once fixed $w_{n-1}, \ldots, w_{0}$.

Next, we choose $n=\lambda$. This means that $w_{n-1}, \ldots, w_{0}$ are constants and as a consequence $p_{n}\left(w_{n}, w_{n-1}, \ldots, w_{0}, H\right)=0$ is an algebraic first integral of the equation (10) satisfied by $w_{n}$.

But the general solution of equation (10) is:

$$
\left\{\begin{array}{l}
w_{n, 1}=-c_{n, 1} / n+2+c_{1} e^{(n+2) s}, \\
w_{n, 2}=c_{n, 2} s+c_{2},
\end{array}\right.
$$

where $c_{1}$ and $c_{2}$ are the free constants of integration. Therefore, equation (10) possesses an algebraic first integral only if $c_{n, 2}=0$ (this first integral is simply $w_{n, 2}=c_{2}$ ), and this implies that the expansions of $x_{1}(t), x_{2}(t)$ are Laurent series.

\section{Non-analytic first integral}

In this section, we are going to establish a theorem about analytic non-integrability for a quadratic system on the plane satisfying hypothesis H1, assuming that $\operatorname{div} D X_{2}(a)$ is an irrational number. In such situation, there exists a particular solution represented in terms of a Laurent series expansion that will allow us to apply a lemma by Ziglin to the variational equations. It is important to notice that a "closed" expression for the particular solution will not be needed.

Theorem 9. Assume that hyphotesis $\mathbf{H 1}$ holds for the quadratic system (1), an that $\lambda:=\operatorname{div} D X_{2}(a)+2$ is not a rational number: $\lambda \notin \mathbb{Q}$. Then,

(i) There exist a solution $\varphi(t)$ of system (1) with a Laurent series expansion:

$$
\varphi(t)=a \tau^{-1}+\sum_{n \geq 0} a_{n} \tau^{n} .
$$

(ii) There exists no non-constant analytic first integral of system (1) in a neighbourhood of $\varphi(t)$.

Proof: From the proof of Theorem 1, as the matrix $\Lambda_{n}=n \operatorname{Id}-\Lambda$ is not a singular matrix for all $n$, it follows readily that there exists a solution $\varphi(t)$ with a Laurent series expansion.

Next, we consider the variational equation of system (1) associated to the solution $\varphi(t)$ :

$$
\dot{v}(t)=D X(\varphi(t)) v,
$$


which takes the form

$$
\dot{v}(t)=\tau^{-1}\left(D X_{2}(a)+\cdots\right) v,
$$

due to hypothesis H1. Next, we perform the change of variables: $v=$ $B w$, as in section 2 , where $B$ is the matrix of eigenvectors of $D X_{2}(a)$ associated to its eigenvalues $-2, \lambda(\lambda \neq-2$ by the hypothesis of an irrational $\lambda$ ).

The equation for $w$ is:

$$
\dot{w}=\tau^{-1}\left[\left(\begin{array}{rr}
-2 & 0 \\
0 & \lambda
\end{array}\right)+\cdots\right] w,
$$

and consequently, we can write the expansion of the components of $w$ as:

$$
w_{1}=\frac{C_{1}}{\tau^{2}}+\cdots, \quad w_{2}=C_{2} \tau^{\lambda}+\cdots,
$$

where the component $w_{1}$ corresponds to the solution $\dot{\varphi}(t)$ of the variational equation (13). Thus, the expansion of $w_{1}$ is a Laurent series.

Now, assume that there exists an analytic non trivial integral of system (1): $G(x)=H$. By using Ziglin lemma (see [Zig82], [Yos87]), there exists a natural $k>0$ such that the following function is a first integral of the variational system (13):

$$
\left.D^{k} G(\varphi(t))\left(v, \ldots{ }^{k}\right), v\right)=C,
$$

or written as a function of the new variables $w$ :

$$
\left.D^{k} G(\varphi(t))\left(B w, \ldots{ }^{k}\right), B w\right)=C .
$$

The expression above is an homogeneous polynomial of degree $k$ in the variables $w_{1}$ and $w_{2}$. If we expand the previous expression as a function of $w_{2}$ and take into account the Laurent series caracter of $w_{1}$, we get:

$$
\gamma_{k}(\tau) w_{2}^{k}+\ldots+\gamma_{1}(\tau) w_{2}+\gamma_{0}(\tau)=C,
$$

where $\gamma_{i}(\tau), i=0, \ldots, k$ are functions of $\tau$ whose expansions only contain integer exponents of $\tau$. From $w_{2}=C_{2} \tau^{\lambda}+O\left(\tau^{\mu}\right), \mu>\lambda$, we get:

$$
\gamma_{k}(\tau)\left(C_{2}^{k} \tau^{\lambda k}+O\left(\tau^{(k-1) \lambda+\mu}\right)\right)+O\left(\tau^{(k-1) \lambda}\right)=C .
$$

The only term with $\tau^{\lambda k}$ in the above expression is $\gamma_{k}(\tau) C_{2}^{k} \tau^{\lambda k}$. So, we deduce $\gamma_{k}(\tau) C_{2}^{k}=0$. We conclude $C_{2}=0$ and we have arrived at a contradiction, since 14 was supposed to be a first integral for all the solutions of the variational equation. It follows that there cannot exist a non-constant analytic first integral in a neighbourbood of $\varphi(t)$. 


\section{An example.}

We consider the following quadratic system:

$$
\left\{\begin{array}{l}
\dot{x}=y+2 \alpha x y \\
\dot{y}=-x+\gamma x^{2}+\beta y^{2}
\end{array}\right.
$$

which corresponds, for $\beta=1-\alpha$, to case II of Table 3 .

The vector $a$ is in this case: $a=\left( \pm \sqrt{(2 \alpha-\beta) /\left(4 \gamma \alpha^{2}\right)},-1 /(2 \alpha)\right)^{\top}$, and the matrix $D X_{2}(a)$ is:

$$
D X_{2}(a)=\left(\begin{array}{cc}
-1 & \pm \sqrt{(2 \alpha-\beta) / \gamma} \\
\pm \sqrt{\gamma(2 \alpha-\beta) / \alpha^{2}} & -\beta / \alpha
\end{array}\right) .
$$

The eigenvalues of $D X_{2}(a)$ are -2 and $\lambda:=1-\beta / \alpha$. So, using the theorem above, if $\beta / \alpha \notin \mathbb{Q}$, there exists a solution $\varphi(t)$ with a Laurent series expansion and the differential system cannot have a non-trivail analytic first integral in a neighbourhood of $\varphi(t)$.

For $\beta=1-\alpha$, the condition for non-existence of an analytic first integral reads as $\alpha \notin \mathbb{Q}$. However, system (15) for $\beta=1-\alpha$ is nothing else but case II of Table 3 which possesses a general first integral. This does not give rise to a contradiction, since the solution $\varphi(t)$ with a Laurent series expansion can be explicitly computed in this case:

$$
\begin{aligned}
\Phi:=\gamma+3 \alpha-1+2(\alpha-1)(2 \alpha-1) & \left(\gamma x^{2}-(3 \alpha-1) y^{2}\right) \\
& -2(\alpha-1)(\gamma+3 \alpha-1) x=0,
\end{aligned}
$$

and the general first integral can be written as:

$$
(1+2 \alpha x)^{\alpha-1} \cdot \Phi^{\alpha}=C,
$$

which, if $\alpha \notin \mathbb{Q}$, is not analytic in a neighbourhood of $\varphi(t)$.

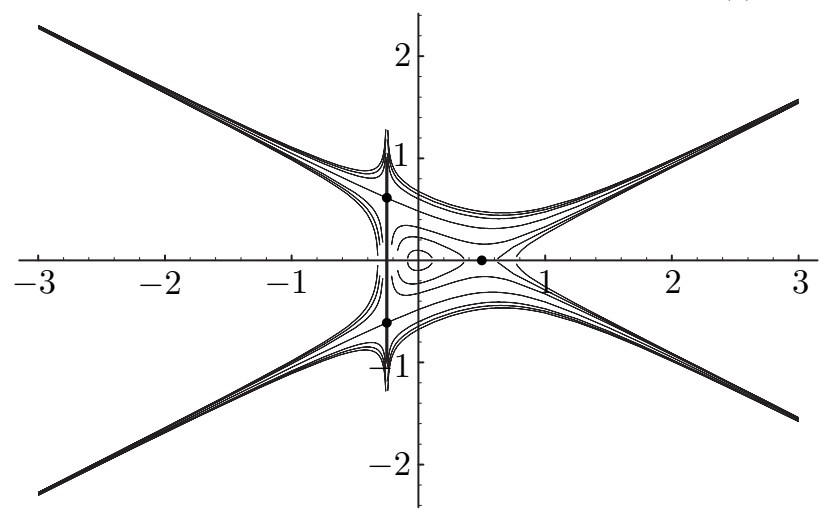

Figure 5. Phase portrait of differential system (15) for parameters $\alpha=2$, $\gamma=2$ and $\beta=1-\alpha=-1$. 
The phase portrait of the integrable system (15) can be seen in Figure 5 for the following values of the parameters: $\alpha=2, \gamma=2$ and $\beta=$ $1-\alpha=-1$. The three dots of the figure are the equilibrium points of the system. The Laurent series solution $\varphi(t)$ corresponds to the separatrix solution passing through two equilibrium points in the line $x=-\frac{1}{4}$ : $\left(-\frac{1}{2 \alpha}, \pm \frac{1}{2 \alpha} \sqrt{\frac{-1-2 \alpha}{\beta}}\right)$.

\section{Proof of Theorem 7}

The proof of this theorem is based on the following lemmas.

Lemma 10. Let us consider the following linear differential system

$$
w_{n}^{\prime}=A_{n} w_{n}+c_{n},
$$

where $A_{n}$ is $m \times m$ matrix with positive eigenvalues and $c_{n}(s)$ is a polynomial vector. Then, the only polynomial solution of system (16) is given by

$$
w_{n}(s)=-\int_{s}^{\infty} e^{(s-u) A_{n}} c_{n}(u) d u=-\sum_{j=0}^{m} A_{n}^{-j-1} c_{n}^{(j)}(s),
$$

where $c_{n}^{(j)}(s)$ is the derivative of order $j$ of $c_{n}(s)$.

Proof: If we use the method of variations of constants, we can write the solution of system (16) as $w_{n}(s)=e^{s A_{n}} U_{n}(s)$, where $U_{n}(s)$ is given by

$$
U_{n}(s)=U_{n}\left(s_{0}\right)+\int_{s_{0}}^{s} e^{-u A_{n}} c_{n}(u) d u .
$$

Since we look for polynomial solutions, $U_{n}(s)$ has to satisfy $\lim _{s \rightarrow \infty} U_{n}(s)=0$ in order to avoid an exponential behaviour at infinity. Taking into account this asymptotic behaviour and using expression (18), we can write $U_{n}\left(s_{0}\right)$ as

$$
U_{n}\left(s_{0}\right)=-\int_{s_{0}}^{\infty} e^{-A_{n} u} c_{n}(u) d u
$$

and we can conclude, using formulas (19) and (18), that the function $w_{n}(s)$ can be written as

$$
w_{n}(s)=-\int_{s}^{\infty} e^{-(s-u) A_{n}} c_{n}(u) d u
$$

This is the first equality of equation (17). Integrating it by parts, we get the second equality. 
Lemma 11. Given $K>0$ and $n>4 \lambda$, let

$$
U(s)=\int_{s}^{\infty} e^{(s-u)(n-\lambda)}(2 K+K u)^{n / \lambda} d u
$$

be a function of the variable $s>0$. Then, $U(s)<\frac{3}{n}(2 K+K s)^{n / \lambda}$.

Proof: Following [Hil74], we introduce $\gamma:=n / \lambda$ and $X:=X(s)=$ $2 K+K s$. Integrating by parts formula (20), we obtain

$$
\begin{aligned}
U(s)<\frac{X^{\gamma}}{n-\lambda}\left(1+\frac{K \gamma}{n-\lambda} X^{-1}\right. & +\frac{K^{2} \gamma(\gamma-1)}{(n-\lambda)^{2}} X^{-2} \\
& \left.+\cdots+\frac{K^{m+1} \gamma(\gamma-1) \cdots(\gamma-m)}{(n-\lambda)^{m+1}}\right)
\end{aligned}
$$

where $m=[\gamma]$. Since $n-\lambda>3 n / 4$, it follows that $(\gamma-j) /(n-\lambda)<1 / \lambda$ and $K / X<1 / 2$ for $j>0$, and so we can deduce that

$$
U(s)<\frac{4}{3 n} X^{\gamma}\left(1+\frac{1}{2 \lambda}+\frac{1}{(2 \lambda)^{2}}+\cdots\right)=\frac{4}{3 n} \frac{2 \lambda}{(2 \lambda-1)} X^{\gamma}<\frac{3}{n} X^{\gamma}
$$

Proof of the Theorem: We only have to prove that the bound

$$
\left\|w_{j}(s)\right\| \leq(j+1)^{-1 / 2}(2 K+K s)^{j / \lambda}
$$

holds for $n \geq 0$. If this is the case, we can conclude that

$$
\left\|\sum_{n=0}^{\infty} w_{n}(s) e^{-n s}\right\| \leq \sum_{n=0}^{\infty}(n+1)^{-1 / 2}(2 K+K s)^{n / \lambda} e^{-n s},
$$

and the theorem follows applying the radical test to the series of the right part.

By hypothesis, bound (21) is valid for $j<\max \left\{4 \lambda, 9 C^{2}+1\right\}$. Proceeding by induction, we assume that the bound (21) is also true for $j<n$, where $n>\max \left\{4 \lambda, 9 C^{2}+1\right\}$.

In particular, $\Lambda_{n}=n \operatorname{Id}-\Lambda$ has positive eigenvalues, and we can use Lemma 10, and write the solution of system (9) as

$$
w_{n}(s)=-\int_{s}^{\infty} e^{(s-u) \Lambda_{n}} c_{n}(u) d u
$$


where

$$
c_{n}(u)=e^{-s} B^{-1} X_{1}\left(B w_{n-1}\right)-\frac{1}{2} B^{-1} \sum_{k=0}^{n-1} D_{2}\left(B w_{k}, B w_{n-k-1}\right) .
$$

As a consequence, we can bound $\left\|w_{n}(s)\right\|$ as

$$
\left\|w_{n}(s)\right\| \leq \int_{s}^{\infty} e^{(s-u)(n-\lambda)}\left\|c_{n}(u)\right\| d u
$$

and it only remains to bound $\left\|c_{n}(u)\right\|$ :

$$
\begin{aligned}
& \left\|c_{n}(u)\right\| \leq e^{-s}\left\|B^{-1}\right\| \cdot\|B\| \cdot\left\|X_{1}\right\| \cdot\left\|w_{n-1}\right\| \\
& \quad+\frac{1}{2}\left\|B^{-1}\right\| \cdot\|B\| \cdot\|B\| \cdot\left\|D_{2}\right\| \sum_{k=0}^{n-1}\left\|w_{k}\right\| \cdot\left\|w_{n-k-1}\right\| \\
& \leq M\left(\frac{\left\|X_{1}\right\|}{\sqrt{n}}+\frac{1}{2}\|B\| \cdot\left\|D_{2}\right\| \sum_{k=0}^{n-1} \frac{1}{\sqrt{k(n-k-1)}}\right)(2 K+K s)^{(n-1) / \lambda} \\
& \leq M\left(\left\|X_{1}\right\|+\frac{\pi}{2}\|B\| \cdot\left\|D_{2}\right\|\right)(2 K+K s)^{(n-1) / \lambda}=C(2 K+K s)^{(n-1) / \lambda} .
\end{aligned}
$$

Using now Lemma 11, we get

$$
\begin{aligned}
\left\|w_{n}(s)\right\| \leq C \int_{s}^{\infty} e^{(s-u)(n-\lambda)}(2 K+K u)^{n / \lambda} & d u \\
& <\frac{3 C}{n}(2 K+K s)^{n / \lambda},
\end{aligned}
$$

and since $n>9 C^{2}+1$, we have $3 C / n<(n+1)^{-1 / 2}$, and we get bound (21) for $j=n$.

The study of the convergence for $s_{c} \in \mathbb{C}$ can be reduced to the real case. Namely, let $s_{c}=s-\mathrm{i} \theta$ be a complex number with $s>0$ and $\theta \in[0,2 \pi)$ (or another interval of length $2 \pi$ ). We perform the following change of time $s: \tau=e^{-s} e^{\mathrm{i} \theta}$, and we notice that $\Im t=\Im t_{p}+|\tau| \sin \theta$.

Substituting this change of time in equation (4), we get

$$
x(s)=a e^{s} e^{-\mathrm{i} \theta}+\sum_{n \geq 0} \bar{p}_{n}(s) e^{-n s},
$$

as a functions in the new variable $s$, where $\bar{p}_{n}$ is related with $p_{n}(s)$ of equation (8) by $\bar{p}_{n}(s)=e^{\mathrm{i} n \theta} p_{n}(s-\mathrm{i} \theta)$. Applying the previous theorem for each $\theta \in[0,2 \pi)$, we get a $K_{\theta}$ where the psi-series are convergent.

Ackowledgements. We are grateful to the referee for several comments and for pointing out to us some references. A. Delshams is partially supported by the Spanish grant DGICYT PB94-0215, the EC grant ERBCHRXCT940460, and the Catalan grant CIRIT 1996SGR-00105. 


\section{References}

[ARS78] M. J. Ablowitz, J. Ramani and H. Segur, Nonlinear evolution equations and ordinary differential equations of Painlevé type, Lett. Nuovo Cimento 23 (1978), 333-337.

[BPB87] T. C. Bountis, V. Papageorgiou and M. Bier, On the singularity analysis of intersecting separatrices in near-integrable dynamical systems, Phys. D 24 (1987), 292-304.

[CC80] Y. F. Chang AND G. CoRliss, Ratio-like and recurrence relation tests for convergence of series, J. Inst. Maths. Applics. 25 (1980), 349-359.

[CF92] L. Cairo and M. R. Feix, Families of invariant of motions for the Lotka-Volterra equations: The linear polynomials family, $J$. Math. Phys. 33(7) (1992), 2440-2455.

[DMS97] A. Delshams, A. Mir and M. T. Seara, Psi-series and splitting of separatrices, Preprint in progress (1997).

[Gav88] L. GaVRILOV, On the non-integrability of a class of differential equations which are not of Painlevé type, C. R. Acad. Bulgare Sci. 41 (1988), 21-24.

[Gav92] L. Gavrilov, Non-integrability of the equations of heavy gyrostat, Compositio Math. 82 (1992), 275-291.

[HCF93] D. D. Hua, L. CAiro and M. R. Feix, Time-independent invariants of motion for the quadratic system, J. Phys. A 26 (1993), 7097-7114.

$\left[\mathrm{HCF}^{+}\right.$96] D. D. Hua, L. Cairo, M. R. Feix, K. S. Govinder AND P. G. L. LEACH, Connection between the existence of first integrals and the painlevé property in two-dimensional lotka-volterra and quadratic systems, Proc. Roy. Soc. London Ser. A 452 (1996), 859-880.

[Hil74] E. L. Hille, A note on quadratic systems, Proc. Roy. Soc. Edinburgh Sect. A $\mathbf{7 2}$ (1974), 17-37.

[Hil76] E. L. HiLLE, "Ordinary Differential Equations in the Complex Domain," Wiley-Interscience, New York, 1976.

[Hor96] J. HoRN, Ueber die reihenentwickelung der integrale eines systems von differentialgleichungen in der umgebung gewisser singulärer stellen, J. Reine Angew. Math. 116 (1896), 265-306.

[Inc56] E. L. InCE, "Ordinary Differential Equations," Dover, New York, 1956.

[LS82] V. A. Lunkevich And K. S. SibirskiI, Integrals of a general quadratic differential systems in cases of a center, Differential 
Equations 18(5) (1982), 563-568.

[MD93] A. Mir and A. Delshams, Singularity analysis for two dimensional systems with rapidly oscillatory forcing, in "International Conference on Differential Equations Equadiff-91," (C. Perelló, C. Simó and J. Solà-Morales, eds.), World Scientific, Singapore, 1993, pp. 754-758.

[MRT95] P. Mardešić, C. Rousseau and B. Toni, Linearization of isochronous centers, J. Differential Equations 121 (1995), 67-108.

[RGB89] A. Ramani, B. Grammaticos and T. Bountis, The Painlevé property and singularity analysis of integrable and nonintegrable systems, Phys. Rev. Lett. 180(3) (1989), 159-245.

[Smi75] R. A. Sмiтh, Singularities of solutions of certain plane autonomous systems, Proc. Roy. Soc. Edinburgh Sect. A 72 (1975), 307-315.

[Yos83] H. Yoshida, Necessary conditions for the existence of algebraic first integrals, I: Condition for algebraic integrability, Celestial Mech. Dynam. Astronom. 31 (1983), 381-399.

[Yos87] H. Yoshida, A criterion for the non-existence of an aditional integral in hamiltonian systems with a homogeneous potential, Phys. D 29 (1987), 128-142.

[Zig82] S. L. ZIGLIN, Branching of solutions and nonexistence of first integrals in Hamiltonian mechanics I, Functional Anal. Appl. 16(3) (1982), 181-189.

Internet access. All the authors' preprints quoted in the references list are available at http://www-ma1.upc.es in the preprints page, or at ftp://ftp-ma1.upc.es, in the pub/preprints directory.

\author{
Amadeu Delshams: \\ Dept. de Matemàtica Aplicada I \\ Universitat Politècnica de Catalunya \\ Diagonal 647 \\ 08028 Barcelona \\ SPAIN \\ e-mail: amadeu@ma1.upc.es
}

\author{
Arnau Mir: \\ Dept. de Matemàtiques i Informàtica \\ Universitat de les Illes Balears \\ Crta. de Valldemossa, Km. 7.5 \\ 07071 Palma de Mallorca \\ SPAIN \\ e-mail:dmiamt0@ps.uib.es
}

Primera versió rebuda el 30 de Novembre de 1996, darrera versió rebuda el 5 de Febrer de 1997 